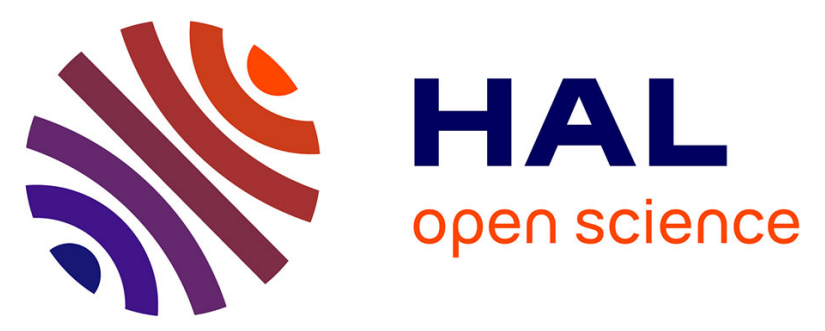

\title{
The Multi Depot One-to-One Pickup and Delivery Problem with Distance Constraints: Real World Application and Heuristic Solution Approach
}

\author{
Olfa Chebbi, Ezzeddine Fatnassi
}

\section{- To cite this version:}

Olfa Chebbi, Ezzeddine Fatnassi. The Multi Depot One-to-One Pickup and Delivery Problem with Distance Constraints: Real World Application and Heuristic Solution Approach. 16th IFIP International Conference on Computer Information Systems and Industrial Management (CISIM), Jun 2017, Bialystok, Poland. pp.391-401, 10.1007/978-3-319-59105-6_33 . hal-01656251

\section{HAL Id: hal-01656251 \\ https://hal.inria.fr/hal-01656251}

Submitted on 5 Dec 2017

HAL is a multi-disciplinary open access archive for the deposit and dissemination of scientific research documents, whether they are published or not. The documents may come from teaching and research institutions in France or abroad, or from public or private research centers.
L'archive ouverte pluridisciplinaire HAL, est destinée au dépôt et à la diffusion de documents scientifiques de niveau recherche, publiés ou non, émanant des établissements d'enseignement et de recherche français ou étrangers, des laboratoires publics ou privés. 


\title{
The multi depot one-to-one pickup and delivery problem with distance constraints: Real world application and Heuristic solution approach
}

\author{
Olfa Chebbi ${ }^{1}$ and Ezzeddine Fatnassi ${ }^{2}$ \\ 1 Université de kairouan, \\ Institut Supérieur d'Informatique et de Gestion de Kairouan, \\ Avenue Khemais El Alouini - 3100 Kairouan Tunisie. \\ 2 Institut Supérieur de Gestion de Tunis \\ Université de Tunis \\ 41, Rue de la Liberté - Bouchoucha - 2000 Bardo, Tunisie
}

\begin{abstract}
This paper presents the development of the multi depot oneto-one pickup and delivery problem with distance constraints problem. This problem involve routing vehicles in a multi depot network topology to satisfy a set of pickup and delivery requests subject to a maximum allowable distance constraint. A problem definition is given and a real world application is proposed for that problem. An approximate solution approach which divides the problem into several subproblems and solve them to optimality is also proposed. Computational experiments show that the proposed solution approach reach good quality solutions is a reasonable computational time.
\end{abstract}

Keywords: Vehicle routing problem, Multi-depot, pickup and delivery requests, transportation problems

\section{Introduction}

Real-life urban on-demand passenger's transportation problems represent a high complex type of problems. In fact, these problems involve a variety of hard constraints which must not been violated. Such constraints involve multi-dimensional vehicle capacity, route duration restriction, time windows, exclusive travels and so on. These problems involve also several complex objective functions which are more complex than the classical distance minimization as several real life factors must be taken into account. However and despite the important interest of these problems, they have received little attention in the literature from an academic point of view.

Generally, real-life urban on-demand passenger's transportation problems are related to the theoretical vehicle routing problems. The vehicle routing problem (VRP) was first proposed by Dantzig and Ramser in 1959 [10]. VRP involves a set of key decisions in order to find a least costs set of customers' sequences to be visited by each vehicle. VRP involves generally several constraints such as distance, capacity, time and cost constraints. 
In the literature, several variants of VRP have been studied which meet and address condition of several real world applications of VRP. One could note for example the capacitated VRP(CVRP)[23], the heterogeneous VRP(HVRP)[8], VRP with time windows, VRP with pickup and delivery (PDP)[26], the multi depot VRP (MDVRP)[19] and so on. For a more clear survey on VRP and its extension, the reader is refereed to [2].

The PDP is the problem where the service required by a customer needs both pickup and delivery service. The PDP can be further subdivided to the many to many PDP, the one to many to one PDP and the one to one PDP. For a more classification of PDP the reader is referred to the survey paper of Berbeglia et al.[2].

The VRP and its several variants such as the PDP and the MDVRP could include several constraints and are known to be NP-Hard problems [2]. The relative complexity of these problem results on a long computational time to find optimal solution especially for large size problems. Consequently, heuristics and metaheuristics approaches have been widely applied and developed to solve large VRP problems. In fact, approximate methods have been able to provide good quality solutions within reasonable computational time.

Based on the works from the literature, relatively new variants of VRP could be proposed to tackle real world application of routing problems. For instance, we could note the works of Sombuntham and Kachitvichyanukul [17] where they proposed a particle swarm optimization algorithm for multi-depot vehicle routing problem with multiple pickup and delivery requests or the multi-depot pickup and delivery problem with a single hub and heterogeneous vehicles [16]. These works proves that the joint study of multiple depot and pickup and delivery problems is an interesting field to develop. However, one could note that none of the recent works in the literature of the multi-depot pickup and delivery problems proposed to add distance constraints related to VRP. Also, one could note that to the best of our knowledge the multi-depot variant of the one-to-one PDP has not been studied in literature before [18][2].

Consequently in this paper, we extends the works in the literature by focusing on the multi-depot one-to-one PDP with distance constraints problem. Starting from an urban on-demand passengers transportation problem related to automated transit system, we motivate the multi depot one-to-one PDP with distance constraints problem. We propose also an adapted linear programming heuristic in order to solve large size instances of the proposed problem in a fast computational time. Finally, the proposed solution approach is proved to be very effective by testing it on a carefully generated set of instances based on our real world context.

Consequently, Section 2 presents the real world application of the multi depot one-to-one PDP with distance constraints. Section 3 develops the problem formulation. Section 4 provides a description of the resolution methodology. Section 5 describes the computational results. Finally, Section 6 concludes the paper. 


\section{The real world application of the multi depot one-to-one PDP with distance constraints}

In this paper, we focus on the multi depot one-to-one PDP with distance constraints by studying its real world urban on-demand passengers transportation application. In fact, there is several transportation systems that are designed to move people in urban areas. Examples of these systems include subway, bus, train, bus rapid transit (BRT), light rapid transit (LRT), automated transit network (ATN) and so on.

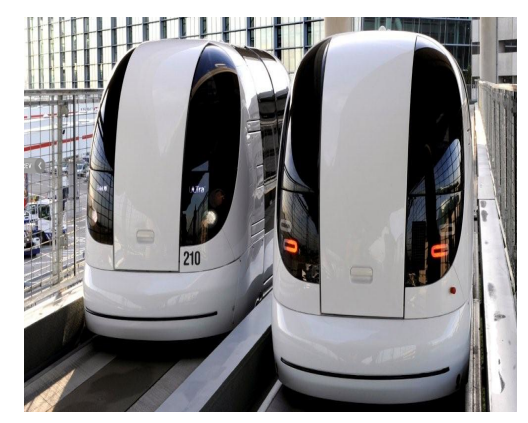

Fig. 1: ATN' vehicles ${ }^{3}$

Among these system, one could focus on the ATN systems. ATN is designed as a public transportation tool to move passengers within urban areas. ATN offers an on-demand non-stop, origin-to-destination transportation service for its users over a dedicated network. ATN uses a set of fully automated vehicles carrying passengers from station to station. ATN'vehicles run on exclusive, gradeseparated set of guideways (see Figure 1). ATN offers an unique transportation service. In fact, ATN'stations are off-line. This feature allows vehicles to travel from origin to destination without any intermediate stops. Furthermore, ATN transportation service is done on-demand where users could choose their time and way of travel. Therefore, ATN offers a taxi-like transportation service. ATN transportation features are quite different from any other automated guideway transit service (AGN) such as LRT, BRT and streetcars.

In the literature, the main features of ATN are defined as follows:

- Small vehicles available for the moving an individual or small group traveling together by choice.

- direct origin-to-destination transportation service without any intermediate stops.

- on demand transportation service.

- fully automated driverless electric vehicles.

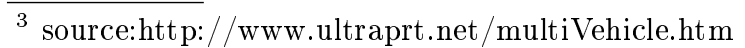


A literature review published in 2005 [9] states that there is more than 200 research papers related to ATN. More recently, several operational and strategic optimization studies related to ATN were published such as network design [25], station design [24], dynamic routing [4][13], simulation [14],[7] energy minimization [22], passengers waiting time [20] total traveled distance [12][15], fleet size[5], optimized operational planning [6][11] and so on.

Starting from all these features, one could note that the transportation service for ATN consists on pickup passengers from a predefined station in order to deliver them to another station. Consequently, the ATN has a strong relation with the PDP problem. Also as the ATN uses a set of driverless vehicles with limited battery capacity, we could found distance constraints related to the routing of ATN vehicles. Finally, as the ATN'network could have multiple depots where initially the ATN vehicles are located, routing vehicles in the ATN context has also a strong relation with the Multi-depot VRP.

However and in the literature, routing problems related to ATN considered only the single depot network topology with different objective such as energy minimization[12], fleet size[5], waiting time of passengers [20] and so on. Consequently and based on all these features and this gap in the literature, we could state that routing driverless electric vehicles in the ATN'network has a strong relation with the multi depot one-to-one PDP with distance constraints.

In the next section, we detail the theoretical problem formulation related to the ATN.

\section{Problem formulation}

In this section, we present the formal problem description and definition of the multi depot one-to-one PDP with distance constraints.

\subsection{Problem description}

Generally in VRPs, a customer is described as a place that must be served by a vehicle from the depot. In this paper, the classic term of customer is replaced by a more general term of "travel". The travel reflects the pickup and delivery transportation service that must be done within the one-to-one PDP. For a PDP problem, a travel is represented by a commodities or a group of persons that must be collected from an origin location and must be delivered to a destination location. As we are treating the one-to-one PDP, the transportation service is done by direct shipment from origin to destination. As each travel has different physical locations, each location can play only one of these three roles: pickup location, delivery location, or depot. Consequently, each location may have several passengers or groups of passengers to be picked up and delivered to several other locations. The transportation situation happening in our problem can be depicted in Figure 2 .

Our problem is based on the following assumptions: 


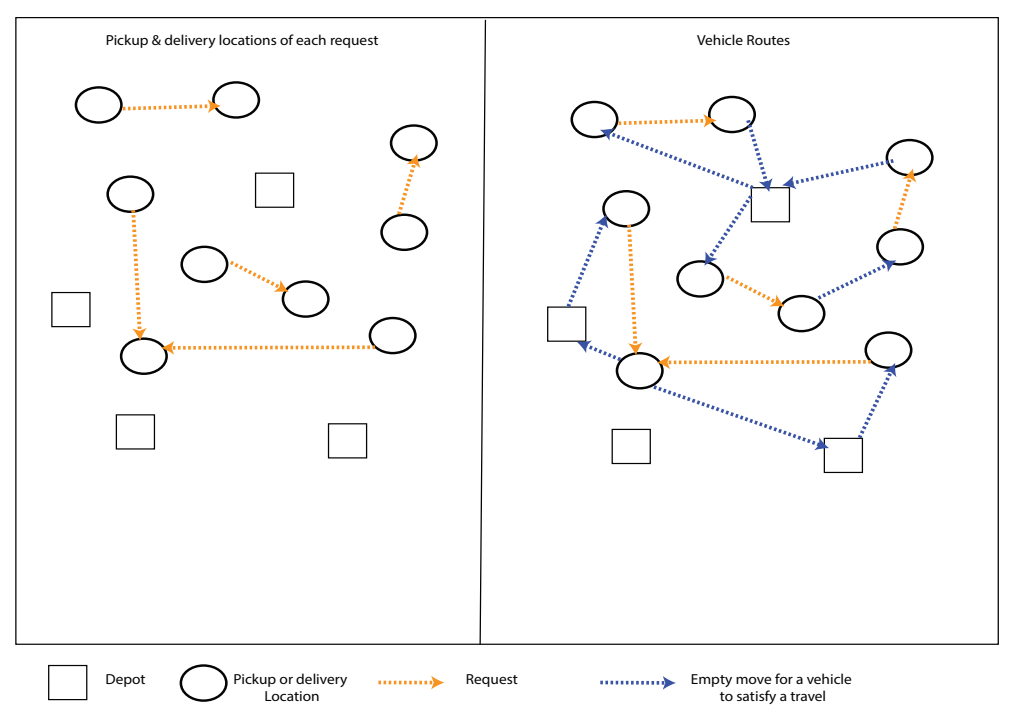

Fig. 2: The VRP with one to one transportation requests.

- Let suppose to have a network composed of $M$ different pickup/delivery location where passengers could embark or disembark from vehicles.

- The network is supposed to be fully connected. A vehicle could move between any pairs of pickup/delivery location.

- Our problem is primarily characterized by a set of travels $T$ known in advance. $|T|=n$.

- Our problem suppose to have also an unlimited number of vehicles. The vehicles are initially located in one of the different depots in the simulated network. The vehicles need to satisfy the transportation demand.

- Each travel contains information concerning transportation demand. In order to satisfy travel $i$, the central control system must assign an empty vehicle in order to satisfy a transportation service from a pickup location $D S_{i}$ to delivery location denoted $A S_{i}$. The service should be provided at the desired departure time, $D T_{i}$, and delays are not permitted. Each travel, $i$, has a specific arrival time $A T_{i}$.

Our objective is to design a set of least-cost journeys that start and end at the depot, are capable of accommodating all travel requests within the allocated time frame and under several constraints such as distance and time window constraints.

Generally, in our problem the vehicles routes have to be constructed in such a way that:

- The total energy consumption is minimized.

- All the travels must be fulfilled. 
In addition, the following restrictions must be met:

- vehicles could serve only one travel at a time.

- Each travel is served by exactly one vehicle.

- The number of routes starting from a depot location must be equal to the number of route ending in the same depot location.

- The total duration of each route does not exceed a preset limit. As in our real world application we are using electric ATN'vehicles with limited electric battery capacity, the total route length must not exceed the maximum allowable travel time by the electric battery.

- Each request must be served exactly at its starting time.

\subsection{Formal Graph Definition of our problem}

More specifically, our problem is based on a non-directed graph $G=\{V, E\}$, where $V=\left\{1,2, \ldots, n, d_{1}, d_{2}, \ldots, d_{k}\right\}$ is the set of vertices in the graph. $d_{1}, d_{2}, \ldots, d_{k}$ represent the different $k$ depots in the network. Let the vertex set $V^{*}=\{1, \ldots, n\}$ corresponds to the set of travels. $E$ represents the set of edges, with $e \in E=$ $\{(i, j) \mid i, j \in V\}$. Note that $E^{*}=\left\{(i, j) \mid i, j \in V^{*}\right\}$.

We add edge $e$ to set $E$ when one of the following conditions is satisfied.

- for each nodes $(i, j) \in V^{*}$, we add an edge if and only if the departure date of travel $j$ exceeds or equals the sum of the arrival time of travel $i$ and the time needed to move from the arrival location of travel $i$ to the departure location of travel $j$. The cost of this edge is the energy used for traveling from the arrival location of travel $i$ to the departure location of travel $j$ and of traveling from the departure location of travel $j$ to the arrival location of travel $j$.

- for each node $i \in V^{*}$ and each depot $d \in D$, an edge $(d, i)$ is added with the cost of moving from $d$ to the departure location of travel $i$ plus the cost of moving from the departure location of travel $i$ to the arrival location of travel $i$.

- we add a final set of edges $(i, d)$ for each node $i \in V^{*}$ and each depot $d \in D$. The cost of these edges includes the cost of moving from the arrival location of travel $i$ to $d$.

\section{The resolution methodology}

In this section, we present our resolution methodology for the problem considered above which we call linear programming heuristic (LPH). Our LPH is based on the principles presented in Algorithm 1.

Based on Algorithm 1, we first solve a relaxed linear program in order to find set of roads starting and ending at one of the different depot in the network. The relaxed linear program founds unfeasible roads as we relaxed the distance constraints. Next our LPH and in order to find feasible roads and therefore feasible solution would use an optimal mathematical model to get feasible roads from the set of unfeasible roads. Next, we present more details of the implemented $\mathrm{LPH}$. 


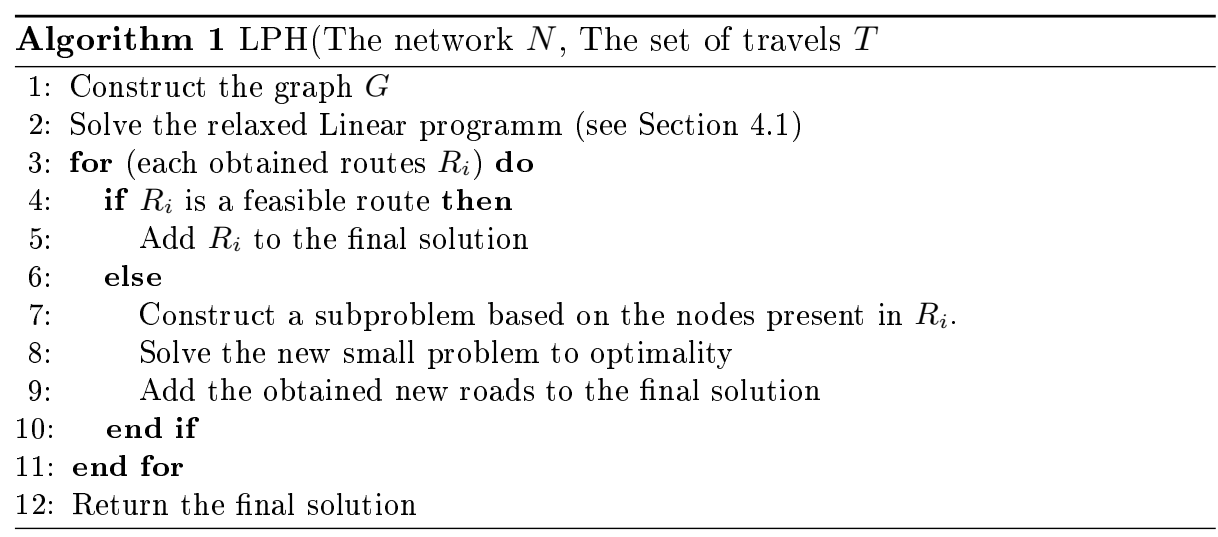

\subsection{The relaxed linear program}

This section presents the relaxed linear program that the LPH will use during its search process.

We first introduce the following integer variable:

$$
x_{i j}=\left\{\begin{array}{l}
1 \text { if } \operatorname{arc}(\mathrm{i}, \mathrm{j}) \text { is selected to match } i \text { to } j \\
0 \text { otherwise }
\end{array}\right.
$$

In our heuristic, we first solve the following relaxed linear program.

$$
\begin{gathered}
: \operatorname{Min} \sum_{(i, j) \in E} c_{i j} x_{i j} \\
\sum_{j \in \delta^{+}(i)} x_{i j}=1 \forall i \in V^{*} \\
\sum_{j \in \delta^{-}(i)} x_{j i}=1 \forall i \in V^{*} \\
\sum_{j \in \delta^{+}(i), k \in \delta^{+}(i)} x_{i j}=\sum x_{k i} \forall i \in D \\
x_{i j} \in\{0,1\} \forall(i, j) \in E
\end{gathered}
$$

Objective function 4.1 minimizes the total energy consumption of the system. Constraints 4.1 and 4.1 ensure that each travel node is served exactly once. Constraints 4.1 ensure that the number of routes starting from a given depot is equal to the number of routes ending in that depot.

However solving this linear program could result on a set of infeasible tour as we do not include maximum distance constraint on it. To fix this dilemma, we propose a specific approach which is presented hereafter. 


\section{$4.2 \quad$ Approach}

Our algorithmic approach is based on the fact that unfeasible routes which violate maximum allowable constraints could be corrected using a valid mathematical formulation of the proposed problem. In fact by solving the relaxed linear program, we could obtain two types of routes: feasible routes and unfeasible routes. Based on our approach, we consider the feasible routes as part of the final solution. The unfeasible routes would be corrected in order to make them respect the maximum allowable distance constraints. In fact from each unfeasible routes, we construct a small routing problem based on its nodes. This small problem would be solved to optimality using a valid mathematical formulation of our problem. This valid mathematical formulation is taken from the works in the literature of Almoustafa et al. [1] while adding the multiple depot constraints to it. Based on these principles, the final feasible solution would be constructed iteratively.

\section{Computational experiments}

We now present the computational study performed to investigate the results of our LPH for solving the proposed optimization problem. An analysis of the obtained results is also performed to prove the efficiency of our algorithm.

\section{$5.1 \quad$ Test Instances}

The proposed algorithm is implemented using the $\mathrm{C}++$ language and tested on a laptop with an Intel(R) Core (TM) i3 CPU M $3802.53 \mathrm{GHz}$ processor and 3 GB RAM (Windows 7 64-bit). Since we are treating a real world application of the GVRP-MDMPDRDC related to the ATN, we based the testing instances on recent works related to ATN [6]. In fact our instance generator is based on the Corby network which represents a real case of routing ATN'vehicles in urban areas. This network represents a realistic case study developed by Bly and Teycheene [3]. Corby is a town in Northampton, (UK) with a population of approximately 50000 . It offers a good example for testing the ATN feasibility in a relatively new town. This network has $14.2 \mathrm{~km}$ of ATN guideways. The Corby network has 15 stations and four depots.

Based on this network, we generated 100 instances where the number of travels varies between 10 and 100 in a steps of 10 . Consequently and for each travel 'size, we generated 10 instances. The list of travels and its characteristics were based on the works from the literature of Mrad and Hidri [22] and Mrad et al. [21]. The maximum allowable time limit for each route was taken to be equal to 40 minutes [6]. We note also that all the mathematical models were solved using IBM-Cplex $12.2^{4}$.

\footnotetext{
${ }^{4}$ Details about Cplex could be found in https://www01.ibm.com/software/commerce/optimization/cplex-optimizer/
} 


\subsection{Computational results}

Results of our method are exposed in Table 1. To assert the quality of the obtained solutions we used the GAP metric. The GAP is obtained as follows:

GAP $=\left(\frac{(S O L-L B)}{L B}\right) \times 100$ We should note that LB is the linear relaxation of the presented mathematical formulation presented in the literature[1].

Table 1 illustrates that the LPH method provides good quality solutions. In fact, the LPH founds an average GAP of $2.435 \%$ in 0.132 seconds. As shown in Table 1, using the LPH method results on obtaining remarkable low GAPs which varies from $0.635 \%$ to $5.075 \%$. Moreover, the GAP with respect to the lower bound values is below $6 \%$. These results mean that the algorithm is capable to converge to a good solution even starting from an unfeasible one which is actually a good point for our algorithm. The good performance of the LPH is further compounded by the fact that it consume small computational time (less than 0.3 seconds). This is due mainly to the straightforward principles that the LPH uses. In fact by solving at each time a relaxed linear program, it has the ability to determine good parts of the final solution quickly.

Table 1: The Obtained Results

\begin{tabular}{l|ll} 
Number of Travels & \multicolumn{2}{|l}{ Average Gap \% Average Time (seconds) } \\
\hline 10 & 0.635 & 0.037 \\
20 & 0.800 & 0.050 \\
30 & 1.649 & 0.067 \\
40 & 1.641 & 0.071 \\
50 & 2.655 & 0.099 \\
60 & 2.682 & 0.124 \\
70 & 3.782 & 0.151 \\
80 & 2.774 & 0.212 \\
90 & 2.655 & 0.234 \\
100 & 5.075 & 0.271 \\
\hline Average & 2.435 & 0.132
\end{tabular}

\section{Conclusions}

The multi depot one-to-one PDP with distance constraints problem has been introduced and formalized in this paper. This is a new VRP that arises in real urban contexts. The goal of this problem is to carry a set of pickup and delivery operations at a minimum costs subject to different constraints related to the time windows and total traveled distance for vehicles. A real world application related to the multi depot one-to-one PDP with distance constraints was proposed. Our application arises in the context of ATN in order to carry a set of passengers from origin to destination locations. The proposed problem was formulated and 
a straightforward linear programming heuristic was proposed to solve it. Computational tests have been carried out on instances with different number of travels while representing realistic network contexts. The results obtained from computational tests show the efficacy and the effectiveness of the proposed approach. The most innovative aspect of this approach is its ability to extract goods part of the final solution while starting from an unfeasible solutions. Future developments in this field could address the introduction of stochastic travel time between locations of the treatment of the proposed routing problem in a dynamic context by the tool of simulation.

\section{References}

1. Almoustafa, S., Hanafi, S., Mladenovi, N.: New exact method for large asymmetric distance-constrained vehicle routing problem. European Journal of Operational Research (2012)

2. Berbeglia, G., Cordeau, J.F., Laporte, G.: Dynamic pickup and delivery problems. European journal of operational research 202(1), 8-15 (2010)

3. Bly, P., Teychenne, R.: Three financial and socio-economic assessments of a personal rapid transit system. In: Proceedings of the tenth international conference on automated people movers. p. 39 (2005)

4. Chebbi, O., Chaouachi, J.: Modeling on-demand transit transportation system using an agent-based approach. In: Computer Information Systems and Industrial Management, pp. 316-326. Springer (2015)

5. Chebbi, O., Chaouachi, J.: Optimal fleet sizing of personal rapid transit system. In: Computer Information Systems and Industrial Management, pp. 327-338. Springer (2015)

6. Chebbi, O., Chaouachi, J.: Reducing the wasted transportation capacity of personal rapid transit systems: An integrated model and multi-objective optimization approach. Transportation Research Part E: Logistics and Transportation Review (2015)

7. Chebbi, O., Chaouachi, J.: Proceedings of the Second International Afro-European Conference for Industrial Advancement AECIA 2015, chap. A Decentralized Management Approach for On-Demand Transit Transportation System, pp. 175-184. Springer International Publishing, Cham (2016), http://dx.doi.org/10.1007/ 978-3-319-29504-6_18

8. Coelho, V., Grasas, A., Ramalhinho, H., Coelho, I., Souza, M., Cruz, R.: An ilsbased algorithm to solve a large-scale real heterogeneous fleet vrp with multi-trips and docking constraints. European Journal of Operational Research 250(2), 367$376(2016)$

9. Cottrell, W.D.: Critical review of the personal rapid transit literature. In: Proceedings of the 10th International Conference on Automated People Movers May. pp. $1-4(2005)$

10. Dantzig, G.B., Ramser, J.H.: The truck dispatching problem. Management science 6(1), 80-91 (1959)

11. Fatnassi, E., Chaouachi, J., Klibi, W.: Planning and operating a shared goods and passengers on-demand rapid transit system for sustainable city-logistics. Transportation Research Part B: Methodological 81, 440-460 (2015) 
12. Fatnassi, E., Chebbi, O., Chaouachi, J.: Discrete honeybee mating optimization algorithm for the routing of battery-operated automated guidance electric vehicles in personal rapid transit systems. Swarm and Evolutionary Computation (2015)

13. Fatnassi, E., Chebbi, O., Siala, J.C.: Evaluation of different vehicle management strategies for the personal rapid transit system. In: Modeling, Simulation and Applied Optimization (ICMSAO), 2013 5th International Conference on. pp. 1-5. IEEE (2013)

14. Fatnassi, E., Chebbi, O., Siala, J.C.: Two strategies for real time empty vehicle redistribution for the personal rapid transit system. In: Intelligent Transportation Systems-(ITSC), 2013 16th International IEEE Conference on. pp. 1888-1893. IEEE (2013)

15. Fatnassi, E., Chebbi, O., Siala, J.C.: Comparison of two mathematical formulations for the offline routing of personal rapid transit system vehicles. In: The International Conference on Methods and Models in Automation and Robotics (2014)

16. Irnich, S.: A multi-depot pickup and delivery problem with a single hub and heterogeneous vehicles. European Journal of Operational Research 122(2), 310-328 (2000)

17. Kachitvichyanukul, V., Sombuntham, P., Kunnapapdeelert, S.: Two solution representations for solving multi-depot vehicle routing problem with multiple pickup and delivery requests via pso. Computers \& Industrial Engineering 89, 125-136 (2015)

18. Lahyani, R., Khemakhem, M., Semet, F.: Rich vehicle routing problems: From a taxonomy to a definition. European Journal of Operational Research 241(1), 1 - 14 (2015), http://ww.sciencedirect.com/science/article/pii/ S0377221714006146

19. Lalla-Ruiz, E., Expósito-Izquierdo, C., Taheripour, S., Voß, S.: An improved formulation for the multi-depot open vehicle routing problem. OR Spectrum 38(1), 175-187 (2016)

20. Lees-Miller, J.D.: Minimising average passenger waiting time in personal rapid transit systems. Annals of Operations Research 236(2), 405-424 (2016)

21. Mrad, M., Chebbi, O., Labidi, M., Louly, M.A.: Synchronous routing for personal rapid transit pods. Journal of Applied Mathematics 2014 (2014)

22. Mrad, M., Hidri, L.: Optimal consumed electric energy while sequencing vehicle trips in a personal rapid transit transportation system. Computers \& Industrial Engineering 79, 1-9 (2015)

23. Prins, C.: A simple and effective evolutionary algorithm for the vehicle routing problem. Computers \& Operations Research 31(12), 1985-2002 (2004)

24. Won, J.M., Choe, H., Karray, F.: Optimal design of personal rapid transit. In: Intelligent Transportation Systems Conference, 2006. ITSC'06. IEEE. pp. 14891494. IEEE (2006)

25. Zheng, H., Peeta, S.: Network design for personal rapid transit under transitoriented development. Transportation Research Part C: Emerging Technologies $55,351-362(2015)$

26. Zhu, Z., Xiao, J., He, S., Ji, Z., Sun, Y.: A multi-objective memetic algorithm based on locality-sensitive hashing for one-to-many-to-one dynamic pickup-and-delivery problem. Information Sciences 329, 73-89 (2016) 